\title{
Recurrent idiopathic pancreatitis in familial adenomatous polyposis: Report of a case-series and review of the literature
}

\author{
Aura A. J. van Esch · Joost P. H. Drenth • \\ Rene H. M. te Morsche · Jan B. M. J. Jansen • \\ Fokko M. Nagengast
}

Received: 17 December 2006/ Accepted: 24 January 2007/Published online: 21 February 2007

(C) Springer Science+Business Media B.V. 2007

\begin{abstract}
Familial adenomatous polyposis (FAP) is characterized by the development of multiple adenomatous polyps predominantly in the colon but also in the duodenum. Scattered case reports indicate that there is a risk for pancreatitis in FAP. The most likely cause of pancreatitis in FAP is obstructing ampullary adenomas. We describe 7 FAP patients who experienced one or more episodes of pancreatitis. Two patients experienced pancreatitis after endoscopic treatment of ampullary adenoma. The cause of the pancreatitis in 5 of 7 patients could not be determined, as none of the patients had obstruction of the ampulla. Furthermore, other risk factors for pancreatitis such as pancreatic serine protease inhibitor Kazal type I (SPINK1) gene mutations were ruled out. A review of literature identified $20 \mathrm{FAP}$ patients who developed the first episode of pancreatitis at a mean age of 45 years (range 23-72 years). Some 55\% had recurrent episodes of pancreatitis. Eight patients had (peri) ampullary adenomas or carcinomas. In most cases, the course of pancreatitis was mild with an uneventful outcome, but one patient died after an episode of acute pancreatitis.
\end{abstract}

Keywords Familial adenomatous polyposis . Pancreatitis · SPINK1

Grant support: Joost P.H. Drenth is a recipient of a NWO-VIDI grant

A. A. J. van Esch $(\varangle)$. J. P. H. Drenth

R. H. M. te Morsche - J. B. M. J. Jansen .

F. M. Nagengast

Department of Gastroenterology and Hepatology, Radboud

University Nijmegen Medical Center, P.O. Box 9101, 6500

HB Nijmegen, The Netherlands

e-mail: A.vanEsch@MDL.umcn.nl

\section{Introduction}

Familial adenomatous polyposis (FAP) is an autosomal dominant inherited disorder, caused by germline mutations in the adenomatous polyposis coli (APC) tumor suppressor gene. Phenotypically, it is characterized predominantly by the development of multiple colonic adenomatous polyps. Apart from colonic adenomas, $58-90 \%$ of FAP patients develop duodenal adenomas, most notably in the proximity of the ampulla. The presence of duodenal adenomas is associated with an approximately 300 times higher risk for duodenal cancer compared to the general population and the lifetime risk is estimated to be $5 \%[1-5]$.

Apart from the presence of colonic and duodenal adenomas, few case reports point to an association of FAP with recurrent acute pancreatitis [6-13]. One reasonable hypothesis is that ampullary adenomas may cause pancreatitis by obstructing the common bile duct [6-13]. On the other hand, not every FAP patient with duodenal adenomatosis will develop pancreatitis, and FAP patients without duodenal adenomas also appear to be at risk for pancreatitis. This suggests that other, as of yet unidentified, factors play a role. Pancreatic serine protease inhibitor, Kazal type I (SPINK1), is a potent inhibitor inhibitor of trypsin activity, and a recent study found that a SPINK1 allele was enriched in a population of patients with acute recurrent pancreatitis [14]. The N34S mutation was present in a frequency of $7.8 \%$ in patients, but only in $2.6 \%$ of healthy controls, which suggests that SPINK1 may play a role in the susceptibility for acute pancreatitis.

The purpose of this study is to report the clinical characteristics of 7 FAP patients who presented with 
acute pancreatitis and to illustrate this condition with an overview of the current literature. In addition, we explored whether SPINK1 mutations contribute to the risk of pancreatitis in these patients.

\section{Materials and methods}

\section{Patients}

The setting of the study was a tertiary referral centre for FAP in the Netherlands. We identified patients by a search of the morbidity database of the department of Gastroenterology and Hepatology, which includes the ICD-9 diagnoses of 114 FAP patients. FAP was diagnosed based upon the presence of more than 100 adenomatous colorectal polyps and a positive family history and/or by genetic testing of an APC mutation. We selected cases with a diagnosis of at least one documented episode of pancreatitis. We reviewed medical records of all cases including results of radiological, endoscopic or pathologic studies. Pancreatitis was diagnosed by clinical abdominal symptoms and elevated serum amylase and defined as idiopathic in case precipitating factors such as alcohol abuse, trauma, medication, infection, metabolic disorders and/or a family history were all absent.

Duodenal adenomas were detected by a side viewing duodenoscope (TJF-160 Olympus) and classified according to the modified Spigelman classification [15]. This classification is based on the number and size of polyps, their histology, and the degree of dysplasia. Stage I and II signify mild duodenal polyposis while stage III and IV indicate severe disease [15].

\section{Literature}

We performed a MEDLINE literature search using the Pubmed interface (http://www.ncbi.nlm.nih.gov/ entrez) for articles focused on patients who had FAP and presented with acute pancreatitis in order to obtain a comprehensive overview of the condition. Our search strategy was performed with the following terms: familial adenomatous polyposis; FAP; acute pancreatitis; duodenal polyposis, for the period 1970-2005.

Articles written in English, French, Italian, Dutch, or German were considered for inclusion in the analysis. The references of the traced articles were scrutinized for additional articles. Data were collected with special attention to the following items: demographic features, age at onset of FAP and pancreatitis, presence and localization of duodenal polyps, comorbidity, and follow-up.

DNA studies

We extracted genomic DNA from whole blood according to established protocols using the Puregene DNA isolation kit (GENTRA systems, Minneapolis, Mn, USA). We searched for two prevalent SPINK1 gene mutations: N34S, and P55S. We sought for these mutations using an allele specific PCR as described elsewhere. Briefly, a $50 \mu \mathrm{l}$ reaction was prepared that contained $200 \mathrm{ng}$ genomic DNA, $10 \mathrm{mM}$ Tris- $\mathrm{HCl}(\mathrm{pH}$ 9.0), $50 \mathrm{mM} \mathrm{KCl}, 0.1 \%$ TRITON, $2 \mathrm{mM} \mathrm{MgCl}$, $0.25 \mathrm{mM}$ dNTP's, $3.0 \mathrm{U}$ Taq-DNA-polymerase and $100 \mathrm{ng}$ of sense, anti sense and mutation primers [16]. Cycling conditions called for an initial step at $94^{\circ} \mathrm{C}$ for $5 \mathrm{~min}$ then 35 cycles at $94^{\circ} \mathrm{C}$ for $30 \mathrm{~s}, 59^{\circ} \mathrm{C}$ for $30 \mathrm{~s}$, $72^{\circ} \mathrm{C}$ for $30 \mathrm{~s}$, and a final elongation step at $72^{\circ} \mathrm{C}$ for $5 \mathrm{~min}$. The PCR product was subjected to gel electrophoresis and analysed by visual inspection.

\section{Results}

We identified 7 FAP patients ( 5 female, 3 male) with a mean age of 44 years (range 21-64 years) who had at least one single episode of acute pancreatitis (Table 1).

\section{Case 1}

A 34-year-old woman (Table 1) with FAP underwent a subtotal colectomy at 17 years of age. She was admitted to the hospital with acute abdominal pain and vomiting. She did not use any medication. Past history included occasional alcohol intake. Laboratory investigations demonstrated an elevated serum amylase (3955 IU/l; normal values <100 IU/l). Other laboratory values including liver enzymes, peripheral blood count, serum calcium and triglyceride levels were within normal range. Ultrasound examination of the abdomen demonstrated signs of acute pancreatitis with fluid surrounding the pancreas, but no abnormalities to the liver or bile duct. A computed tomography (CT) revealed slight swelling of the pancreas and some infiltration in surrounding areas. Acute pancreatitis was diagnosed and treated conservatively. Because of an elevation of temperature and infection parameters antibiotics were added, although the CT did not show any signs of necrosis or infection. She recovered well and after 7 days she was discharged from the hospital. She had two recurrences of pancreatitis, which were treated con- 
Table 1 Clinical data of the 7 FAP-patients from the Radboud University Medical Center

\begin{tabular}{|c|c|c|c|c|c|c|c|}
\hline Patients & 1 & 2 & 3 & 4 & $5^{\mathrm{a}}$ & 6 & 7 \\
\hline Sex & $\mathrm{M}$ & $\mathrm{F}$ & $\mathrm{F}$ & $\mathrm{F}$ & $\mathrm{F}$ & $\mathrm{F}$ & $\mathrm{M}$ \\
\hline Date of birth & 1962 & 1952 & 1972 & 1975 & 1971 & 1953 & 1942 \\
\hline $\begin{array}{l}\text { Age at diagnosis FAP } \\
\text { (years) }\end{array}$ & 18 & 31 & 28 & 20 & 17 & 24 & 42 \\
\hline $\begin{array}{l}\text { Age at diagnosis } \\
\text { duodenal adenomatosis } \\
\text { (years) }\end{array}$ & 33 & 53 & 28 & 30 & 30 & 44 & 55 \\
\hline $\begin{array}{l}\text { Age at diagnosis } \\
\text { pancreatitis (years) }\end{array}$ & 22 & 51 & 33 & 26 & 35 & 50 & 60 \\
\hline Spigelman classification & II & II & III & II & II & II & III \\
\hline $\begin{array}{l}\mathrm{N}^{\circ} \text { of episodes } \\
\text { pancreatitis }\end{array}$ & $>5^{\mathrm{b}}$ & 1 & 2 & 2 & 3 & $1^{\mathrm{c}}$ & $1^{\mathrm{d}}$ \\
\hline $\begin{array}{l}\text { SPINK1 mutation N34S / } \\
\text { P55S }\end{array}$ & Absent & Absent & Absent & Absent & Absent & Not tested & Not tested \\
\hline MRPC/ERCP & Normal & $\mathrm{ND}^{\mathrm{e}}$ & ND & ND & Normal & ND & $\begin{array}{l}\text { ECRP with } \\
\text { papillectomy }\end{array}$ \\
\hline Relevant comorbidity & - & $\begin{array}{l}\text { Hyper- } \\
\text { parathyroidism }\end{array}$ & - & - & - & - & - \\
\hline $\begin{array}{l}\text { Interventions prior to } \\
\text { pancreatitis }\end{array}$ & - & - & - & - & - & $\begin{array}{l}\text { APC of } \\
\text { ampullary } \\
\text { adenoma. }\end{array}$ & $\begin{array}{l}\text { Endoscopic } \\
\text { papillectomy }\end{array}$ \\
\hline
\end{tabular}

a described patient

b resulting in chronic pancreatitis

c patient developed pancreatitis after endoscopic laser treatment of an ampullary adenoma

d patient developed pancreatitis after ERCP with papillectomy because of ampullary adenoma with high grade dysplasia

e ND: not done

f APC: Argon Plasma Coagulation

servatively and the subsequent course was uneventful so far. The etiology of the recurrent episodes of pancreatitis could not be determined. Alcohol abuse was ruled out, and we failed to elicit a positive family history for the disease. Duodenoscopy showed approximately five small duodenal polyps, none in the proximity of the ampulla, classified as Spigelman stage II. Histological analysis showed only low-grade dysplasia. Magnetic resonance imaging (MRI-MRCP) revealed no abnormalities of the pancreatic ducts and no signs of gallstones.

Molecular analysis demonstrated that she possessed two wild type copies of the SPINK1 gene, excluding the N34S and P55S mutations.

\section{Case series}

Table 1 summarizes the pertinent clinical data of all FAP patients who had at least one single episode of acute pancreatitis. Two patients experienced pancreatitis after endoscopic treatment of an ampullary adenoma. In one patient endoscopic snare papillectomy was performed because of an ampullary adenoma with high-grade dysplasia. Immediately after the procedure he developed pancreatitis, which was treated conservatively and resolved without sequelae. Another patient experienced pancreatitis after endoscopic plasma coagulation treatment of an ampullary adenoma. This was treated conservatively and the subsequent course was uneventful. She underwent a surgical papillectomy 1-year later uneventful.

We could exclude obvious causes for acute pancreatitis in all other cases. All patients have duodenal polyps, but there were no signs of obstruction of the ampulla. Gallstones either in the gallbladder or in the common bile duct were absent. Furthermore, there was no history of excessive alcohol consumption. At the time of pancreatitis, the patients did not use drugs associated with pancreatitis.

One patient had concomitant hyperparathyroidism, with marginally elevated serum calcium concentrations (Calcium $2.73 \mathrm{mmol} / \mathrm{l}$, albumin $32 \mathrm{~g} / \mathrm{l}$ ). Although hyperparathyroidism may be associated with acute pancreatitis, it is debatable whether it contributed to the development of this episode of pancreatitis. In most instances it occurs when hypercalcaemia is moderate to severe or if concomitant risk factors such as treatment with steroids and azathioprin are present. One patient had recurrent episodes of acute pancreatitis resulting in chronic 
pancreatitis, while pancreatitis resolved without sequelae in the remaining 4 patients. Genetic analysis of the SPINK1 gene showed no mutation in all cases. The patients with single bouts of pancreatitis after endoscopic treatment of ampullary adenoma were not genetically tested.

\section{Literature search}

We identified eight articles describing patients with FAP and pancreatitis [6-13]. (Table 2) Most articles were case reports on one to three cases $[6,8-12)$. Two articles described a series of patients [7, 13]. In a series of 141 patients with FAP, 5 patients with pancreatitis were reported [7]. The total population of patients with pancreatitis from all articles combined consisted of 21 patients (13 female, 4 male, 4 unknown). All patients were diagnosed with FAP and underwent colectomy. The mean age of onset of pancreatitis was 45 years (range 23-72 years). The oldest patient ( 72 years) was diagnosed with pancreatitis after biopsies of a papillary polyp. A total of 12 of 20 patients experienced recurrent episodes of pancreatitis. The majority of patients were subjected to endoscopic retrograde cholangiopancreaticography (ERCP). Three patients had a peri-ampullary adenocarcinoma and 5 patients had an ampullary adenoma. Six patients had duodenal adenomas. Snare resection of the ampullary adenoma and subsequent sphincterotomy was performed in 4 patients. Sixteen patients underwent pancreatic surgery. The performed procedures ranged from drainage of a pancreatic abscess to Whipple's resection and Roux-Y choledochojejunostomy. Eight patients eventually underwent a pancreatectomy or pancreaticoduodenectomy was performed. In most cases, the outcome was uneventful. Follow-up was not reported in all cases. One patient died after an episode of acute pancreatitis.

\section{Discussion}

This report describes 7 patients from one centre who developed acute pancreatitis in the context of FAP. Pancreatitis is a severe and potentially lethal complication in FAP patients and may contribute to the extracolonic causes of morbidity and mortality in FAP. Acute pancreatitis in itself is a rare event and it has been estimated that the incidence of acute pancreatitis (of all causes) lies around 5-10 per 100.000 (0.005$0.01 \%$ ) in Western Europe [17]. Our data along with data from the literature suggests that the risk for pancreatitis in FAP higher than that, and one series reported a frequency of pancreatitis in FAP of $3.5 \%$ (5/141) [7]. This suggests that pancreatitis is part of the extracolonic phenotype of FAP. Few aspects can be gleaned from literature review. First, the first episode of pancreatitis occurs at middle age, mean 45 years; range 23-72 years. Second, it appears that the majority of patients will have a recurrence of their pancreatitis. Further, 8 of 21 patients had (peri) ampullary adenomas that might or might not have contributed to the pancreatitis. Lastly, although outcome was uneventful in most cases, one patient died after an episode of acute pancreatitis.

There appear to be a few major causes for pancreatitis in FAP patients. First, pancreatitis that arises from obstruction of the pancreatic duct/common bile duct because of ampullary adenomas. Duodenal adenomas are a common manifestation of FAP and it is estimated that $54 \%$ of FAP patients will develop duodenal adenomas twenty years after colectomy while at 75 -years-of-age the prevalence may reach $98 \%$ $[3,4,18]$. Advanced periampullary adenomas are seen in $20 \%$ of patients by 60 -years-of-age [18]. Duodenal adenomas are not usually associated with symptoms, but there is an increased risk for development of duodenal and especially ampullary cancer. The incidence of duodenal cancer in FAP may reach $4 \%$, which is 100-300 times higher than the background population [2]. The high risk for malignant degeneration underscores the necessity for screening, most preferably using side-viewing endoscopes. Lastly, we identified a sample of 5 of 7 patients in whom no clear risk factor was apparent. We carefully searched for underlying risk factors but none of the patients had obstructing ampullary adenomas. Furthermore, other common risk factors for pancreatitis were absent. This contrasts the common dogma that FAP related pancreatitis is caused by obstruction by ampullary adenomas. We therefore sought for other host factors that might explain why these patients are at high risk for the development of acute pancreatitis. As a recent study demonstrated that recurrent idiopathic pancreatitis might be associated with a SPINK1 gene mutation, we searched for two of the most common SPINK1 gene mutations (N34S/P55S), but failed to detect them in our sample. Although this does not rule out that SPINK1 plays a role in FAP associated pancreatitis, it makes it less likely.

In conclusion, we report 7 FAP patients who presented with acute (recurrent) idiopathic pancreatitis without the presence of obstructing ampullary adenomas. This suggests that pancreatitis may be a manifestation of FAP although the actual mechanism is unclear. 


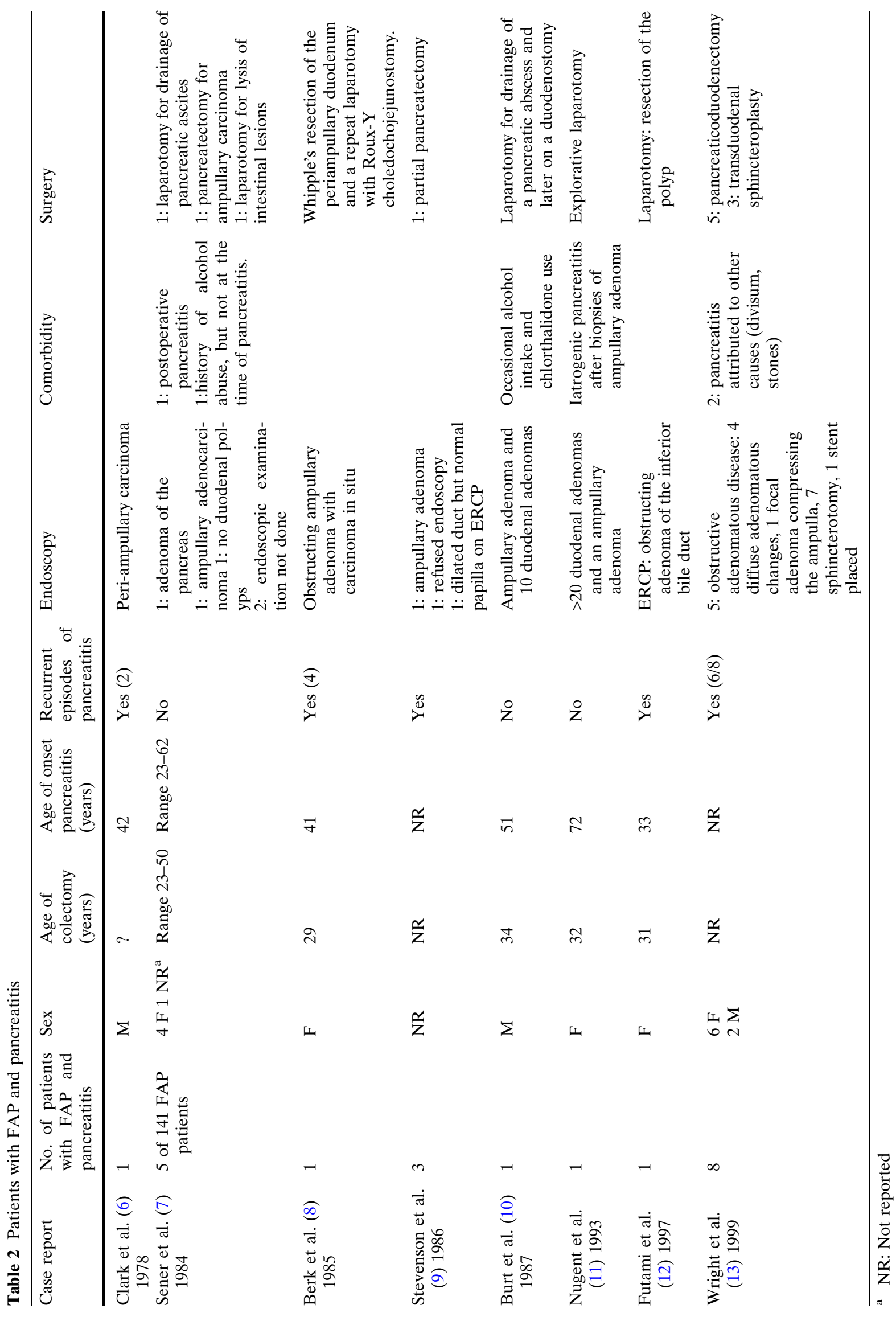




\section{References}

1. Offerhaus GJ, Giardiello FM, Krush AJ et al (1992) The risk of upper gastrointestinal cancer in familial adenomatous polyposis. Gastroenterology 102:1980-1982

2. Kadmon M, Tandara A, Herfarth C (2001) Duodenal adenomatosis in familial adenomatous polyposis coli. A review of the literature and results from the Heidelberg Polyposis Register. Int J Colorectal Dis 16:63-75

3. Bulow S, Bjork J, Christensen IJ et al (2004) Duodenal adenomatosis in familial adenomatous polyposis. Gut 53:381-386

4. Church JM, McGannon E, Hull-Boiner S et al (1992) Gastroduodenal polyps in patients with familial adenomatous polyposis. Dis Colon Rectum 35:1170-1173

5. Stolte M, Pscherer C (1996) Adenoma-carcinoma sequence in the papilla of Vater. Scand J Gastroenterol 31:376-382

6. Clarke DN, Smith JA, Norman JN et al (1978) Pancreatitis and duodenal obstruction due to periampullary carcinoma associated with familial polyposis coli. Postgrad Med J 54:418-420

7. Sener SF, Miller HH, DeCosse JJ (1984) The spectrum of polyposis. Surg Gynecol Obstet 159:525-532

8. Berk T, Friedman LS, Goldstein SD et al (1985) Relapsing acute pancreatitis as the presenting manifestation of an ampullary neoplasm in a patient with familial polyposis coli. Am J Gastroenterol 80:627-629

9. Stevenson JK, Reid BJ (1986) Unfamiliar aspects of familial polyposis coli. Am J Surg 152:81-86

10. Burt RW, Rikkers LF, Gardner EJ et al (1987) Villous adenoma of the duodenal papilla presenting as necrotizing pancreatitis in a patient with Gardner's syndrome. Gastroenterology 92:532-535

11. Nugent KP, Spigelman AD, Williams CB et al (1993) Iatrogenic pancreatitis in familial adenomatous polyposis. Gut 34:1269-1270

12. Futami H, Furuta T, Hanai $\mathrm{H}$ et al (1997) Adenoma of the common human bile duct in Gardner's syndrome may cause relapsing acute pancreatitis. J Gastroenterol 32:558-561

13. Wright BE, Kozarek RA, Traverso LW et al (1999) Recurrent pancreatitis in Gardner variant familial polyposis: etiology, diagnostic approach, and interventional results. Arch Surg 134:311-315

14. Tukiainen E, Kylanpaa ML, Kemppainen E et al (2005) Pancreatic secretory trypsin inhibitor (SPINK1) gene mutations in patients with acute pancreatitis. Pancreas 30:239-242

15. Spigelman AD, Williams CB, Talbot IC et al (1989) Upper gastrointestinal cancer in patients with familial adenomatous polyposis. Lancet 30(2):783-785

16. Drenth JP, te Morsche R, Jansen JB (2002) Mutations in serine protease inhibitor Kazal type 1 are strongly associated with chronic pancreatitis. Gut 50:687-692

17. Banks PA (2002) Epidemiology, natural history, and predictors of disease outcome in acute and chronic pancreatitis. Gastrointest Endosc 56(6 Suppl):S226-S230

18. Bjork J, Akerbrant H, Iselius L et al (2001) Periampullary adenomas and adenocarcinomas in familial adenomatous polyposis: cumulative risks and APC gene mutations. Gastroenterology 121:1127-35 